\title{
Biolubrifiants et écolabel européen
}

François VAN DIEVOET

BfB Oil Research SA,

Parc scientifique Crealys,

rue Phocas Lejeune 10,

B-5032 Gembloux,

France

<bfb@proximedia.be>

Depuis janvier 2005, l'Europe dispose enfin d'un écolabel consacré aux lubrifiants. Celui-ci a été établi sous l'égide de l'Ivam par un groupe de travail constitué de représentants de l'industrie des lubrifiants, de producteurs de matériel et des représentants des ministères de l'environnement de l'Europe des $15 \quad$ (25 aujourd'hui).

L'écolabel européen est largement inspiré des écolabels nationaux existant en Allemagne, " Ange Bleu », qui peut être considéré comme étant le précurseur, et l'écolabel scandinave «White Swan ».

Cet écolabel, dans un premier temps, s'adresse aux lubrifiants suivants :

- fluides hydrauliques;

- graisses;

- huiles de chaînes de tronçonneuses et autres lubrifiants à usage perdu (par exemple : huiles de démoulage);

- huiles pour moteurs deux-temps.

L'écolabel européen fixe non seulement les critères environnementaux, mais aussi les exigences techniques que doivent satisfaire les lubrifiants. D'autre part, une utilisation minimale d'huile de base d'origine renouvelable est prévue.

L'écolabel européen est inscrit depuis janvier 2005 dans le règlement CE 1980/2000: les lubrifiants ont été attribués au groupe 27 (voir le texte donné ci-après).

L'écolabel a une validité de quatre ans et le candidat à l'obtention d'un écolabel doit présenter le dossier à l'autorité compétente de son pays d'origine.

Les adresses figurent sur le site Internet de la CEE :

http://www.europa.eu.int/comm/environment/ ecolabel/product/pg_lubricants_en.htm

En ce qui concerne l'écolabel allemand dit "Ange Bleu », celui-ci a été revu en 2000. ॥ définit les critères environnementaux des huiles de base et des additifs utilisés dans la formule. Ces critères ont servi de base à l'élaboration de l'écolabel européen et sont donc très proches, avec cependant, en ce qui concerne les essais

\begin{abstract}
Text of EEC Commission of 26 april 2005 (2005/360/CE) establishing an Ecolabel for a number of lubricants. Overview and description of the 7 criteria's fixing the new ecolabel.
\end{abstract}

Key words: Europe, ecolabel, biodegradability, ecotoxicity, performances

d'écotoxicité, un test d'inhibition des bactéries et un test de toxicité sur les plantes selon la méthode OECD 208.

L'écolabel scandinave "Cygne Blanc " participe au même principe que I'«Ange Bleu», avec de plus une exigence qui a été reprise dans l'écolabel européen en ce qui concerne la teneur en matières d'origine renouvelable. La mise à disposition aux autorités d'un écolabel concernant les environnements les plus « sensibles » devrait permettre la mise en place de règles et de lois obligeant l'utilisation de ce type de lubrifiants.

L'industrie productrice de lubrifiants possède dans sa gamme les produits adéquats. Encore faut-il inciter les consommateurs à en faire usage dans l'intérêt de tous.

Décision de la Commission du 26 avril 2005 établissant les critères écologiques et les exigences associées en matière d'évaluation et de vérification pour l'attribution du label écologique communautaire aux lubrifiants [notifiée sous le numéro C(2005) 1372]

(2005/360/CE)

La Commission des Communautés Européennes,

- vu le traité instituant la Communauté européenne ;

- vu le règlement (CE) no 1980/2000 du Parlement européen et du Conseil du 17 juillet 2000 établissant un système communautaire révisé $d^{\prime}$ attribution du label écologique ${ }^{1}$, et notamment son article 6 , paragraphe 1 , second alinéa, après consultation du comité de l'Union européenne pour le label écologique, considérant ce qui suit :

(1) En vertu du règlement (CE) no 1980/2000, le label écologique communautaire peut être attribué à un produit présentant des caractéristiques qui lui permettent de contribuer de manière significative à l'amélioration d'aspects environnementaux essentiels.

$\overline{{ }^{1}(1) \text { JO L } 237}$ du 21.9.2000, p. 1.
(2) Le règlement (CE) no 1980/2000 dispose que des critères spécifiques du label écologique, inspirés des critères définis par le comité de l'Union européenne pour le label écologique, doivent être établis par catégories de produits.

(3) Comme l'utilisation de lubrifiants peut être dangereuse pour l'environnement, du fait de leur toxicité aquatique ou de leur bioaccumulation par exemple, il convient d'établir des critères écologiques appropriés.

(4) L'impact environnemental peut être considéré comme négligeable dans le cas des substances contenues dans les lubrifiants dont la nature chimique est modifiée lors de l'application et ne doivent plus être classées conformément à la directive 1999/45/CE du Parlement européen et du Conseil du 31 mai 1999 concernant le rapprochement des dispositions législatives, réglementaires et administratives des États membres relatives à la classification, à l'emballage et à l'étiquetage des préparations dangereuses ${ }^{2}$. Les critères des labels écologiques ne doivent donc pas s'appliquer aux substances dont moins de 0,1\% subsiste sous la forme observée antérieurement à l'application sur l'élément traité.

(5) Les critères écologiques et les exigences associées en matière d'évaluation et de vérification doivent être valables pendant une période de quatre ans.

(6) Les mesures prévues par la présente décision sont conformes à l'avis du comité institué par l'article 17 du règlement (CE) no 1980/2000,

\section{A arrêté la présente décision :}

\section{Article premier}

La catégorie de produits «lubrifiants» comprend les huiles hydrauliques, graisses, huiles pour scies à chaîne, huiles pour moteur à deux temps, agents de décoffrage du béton et autres

$\overline{{ }^{2}(2) ~ J O ~ L ~} 200$ du 30.7.1999, p. 1. 
produits de graissage d'appoint, à usage professionnel et privé.

\section{Article 2}

1. Aux fins de la présente décision, on entend par :

a) « lubrifiant », une préparation composée de fluides de base et d'additifs; L 118/26 FR Journal officiel de I'Union européenne 5.5.2005 la directive 2004/66/CE du Conseil (JO L 168 du 1.5.2004, p. 35) ;

b) « fluide de base », un fluide lubrifiant dont l'écoulement, le vieillissement, l'onctuosité et les propriétés antiusure ainsi que les propriétés de maintien en suspension des polluants $n$ 'ont pas été améliorés par l'ajout d'additifs ;

c) " épaississant », une substance dans le fluide de base destinée à épaissir ou à modifier la rhéologie d'un fluide lubrifiant ou d'une graisse ;

d) «composant principal ", toute substance représentant plus de $5 \%$ en poids du lubrifiant ;

e) "additif ", une substance essentiellement destinée à améliorer l'écoulement, le vieillissement, l'onctuosité, les propriétés antiusure ou de maintien en suspension des polluants ;

f) " graisse », une préparation solide ou semisolide obtenue par dispersion d'un agent épaississant dans un lubrifiant liquide.

2. Dans le cas des graisses, d'autres composants conférant des propriétés particulières peuvent être ajoutés.

\section{Article 3}

Pour pouvoir obtenir le label écologique communautaire pour les lubrifiants au titre du règlement (CE) no 1980/2000, un lubrifiant doit appartenir à la catégorie de produits «lubrifiants» et doit satisfaire aux critères énoncés à l'annexe de la présente décision.

Les critères s'appliquent aux produits de fabrication récente au moment de la livraison. Lorsque des critères portent sur les composants, ils s'appliquent à toute substance qui a été volontairement ajoutée et représente plus de 0,1\% de la composition du produit, mesuré avant et après toute réaction chimique entre les substances mélangées pour obtenir la préparation lubrifiante.

Toutefois, les critères ne s'appliquent pas aux substances dont la nature chimique est modifiée lors de l'application de telle sorte que le classement conformément à la directive 1999/45/CE ne se justifie plus, et dont moins de $0,1 \%$ subsiste sous la forme antérieure à l'application sur l'élément traité.

\section{Article 4}

Les critères écologiques correspondant à la catégorie de produits «lubrifiants», ainsi que les exigences associées en matière d'évaluation et de vérification, sont valables jusqu'au 31 mai 2009.

Article 5

Le numéro de code attribué à des fins administratives à la catégorie de produits «lubrifiants» est «27».

Article 6

Les États membres sont destinataires de la présente décision.

Fait à Bruxelles, le 26 avril 2005.

Par la Commission

Stavros DIMAS

Membre de la Commission

\section{Annexe}

\section{Principe}

\section{Finalité des critères}

Ces critères visent en particulier à promouvoir les produits qui :

- sont peu nocifs pour l'eau et les sols au cours de leur utilisation, et

- permettent de réduire les émissions de $\mathrm{CO}_{2}$.

\section{Exigences en matière d'évaluation et de verification}

Les exigences spécifiques en matière d'évaluation et de vérification sont indiquées pour chaque critère. Lorsqu'il est demandé au demandeur de fournir à l'organisme compétent des déclarations, documents, analyses, comptes rendus d'essai ou tout autre élément attestant la conformité avec les critères, il est entendu qu'ils peuvent être fournis par le demandeur et/ou, le cas échéant, par son (ses) fournisseur(s) et/ou par le(s) fournisseur(s) de ceux-ci, etc. Le fournisseur de l'additif, de l'ensemble d'additifs ou du fluide de base peut fournir les informations pertinentes directement à l'organisme compétent. Au besoin, des méthodes d'essai autres que celles indiquées pour chaque critère peuvent être utilisées si l'organisme compétent qui examine la demande estime qu'elles sont équivalentes. Si besoin est, les organismes compétents peuvent exiger des documents complémentaires et effectuer des contrôles indépendants. II est recommandé aux organismes compétents de tenir compte de l'application de systèmes reconnus de gestion environnementale, tels que EMAS ou ISO 14001, lorsqu'ils évaluent les demandes et vérifient que le produit respecte les critères.

(Remarque: la mise en œuvre de ces systèmes de gestion n'est pas obligatoire.)

\section{Critères}

\section{Phrases de risque indiquant un danger pour I'environnement et la santé humaine}

Aucune phrase de risque indiquant un danger pour l'environnement et la santé humaine, conformément à la directive 1999/45/CE, ne doit s'appliquer au produit au moment de la demande de label écologique. Pour cette catégorie de produits, sont considérées comme pertinentes les phrases de risque suivantes: $R$ $20, R 21, R 22, R 23, R 24, R 25, R 26, R 27, R 28$, $R 33, R 34, R 35, R 36, R 37, R 38, R 39, R 40, R$ $41, R 42, R 43, R 45, R 46, R 48, R 49, R 50, R 51$, R 52, R 53, R 59, R 60, R 61, R 62, R 63, R 64, R $65, R 66, R 67, R$ 68, et leurs combinaisons.

\section{Évaluation et vérification du critère 1}

La conformité avec le critère 1 doit être établie par écrit à l'aide d'un document signé par l'entreprise demanderesse.

II convient de déclarer tous les composants principaux du produit avec précision, en donnant leur nom et, le cas échéant, leur numéro Einecs ou Elincs et les concentrations auxquelles ils sont utilisés.

Le fabricant du produit doit fournir à l'organisme compétent :

- une fiche de données de sécurité du produit [satisfaisant aux exigences de la directive 91/155/CEE de la Commission ${ }^{3}$;

- des fiches de données de sécurité de la part des fournisseurs demandeurs [satisfaisant aux exigences de la directive 91/155/CEE et de la directive 67/548/CEE du Conseil ${ }^{4}$ (2)] pour chaque composant principal.

L 118/28 FR Journal officiel de I'Union européenne 5.5.2005

II convient de fournir suffisamment de données pour permettre l'évaluation des dangers que présente le produit pour l'environnement (indiqués par les phrases de risque: $\mathrm{R} 50, \mathrm{R}$ $50 / 53$, R 51/53, R 52, R 52/53, R 53) conformément aux directives 91/155/CEE et 1999/45/CE.

L'évaluation des dangers que présente le produit pour l'environnement doit être réalisée selon la méthode conventionnelle indiquée à I'annexe III de la directive 1999/45/CE. Toutefois, comme précisé à la partie $\mathrm{C}$ de l'annexe III de ladite directive, le résultat des essais sur la préparation (qu'il s'agisse du produit ou de l'ensemble d'additifs) peut, en tant que tel, modifier la classification relative à la toxicité aquatique qui aurait été obtenue par la méthode conventionnelle.

\section{Exigences supplémentaires en matière de toxicité aquatique}

Le demandeur doit attester la conformité en satisfaisant aux exigences du critère 2.1 ou du critère 2.2.

\section{Critère 2.1}

Exigences concernant la préparation et ses composants principaux

\footnotetext{
$\overline{3}$ JO L 76 du 22.3 .1991$, p. 35

${ }^{4}$ JO 196 du 16.8.1967, p. 1
} 
Doivent être fournies des données sur la toxicité aquatique:

- de la préparation, et

- des composants principaux.

La concentration critique pour la toxicité aquatique de chaque composant principal doit être de $100 \mathrm{mg} / \mathrm{L}$ au minimum.

L'essai doit être effectué sur des algues et des daphnies (OCDE 201 et 202).

La concentration critique pour la toxicité aquatique des huiles hydrauliques doit être de 100 $\mathrm{mg} / \mathrm{L}$ au minimum.

La concentration critique pour la toxicité aquatique des graisses, huiles pour scies à chaîne, agents de décoffrage du béton et autres produits de graissage d'appoint doit être de 1000 $\mathrm{mg} / \mathrm{L}$ au minimum.

II est possible de procéder à l'évaluation des graisses en fournissant seulement des données pour la préparation et les composants principaux si l'épaississant présente une biodégradabilité ultime (voir critère 3 ) ou une biodégradabilité intrinsèque répondant aux conditions suivantes:

- biodégradation > $70 \%$ lors de l'essai OCDE $302 \mathrm{C}$ de biodégradabilité intrinsèque ou selon des méthodes d'essai équivalentes, ou ;

- biodégradation $>20 \%$ mais $<60 \%$ après vingt-huit jours lors des essais OCDE 301 fondés sur la déperdition d'oxygène ou la production de gaz carbonique, ou ;
- biodégradation $>60 \%$ lors de l'essai ISO 14593 («essai au CO2 dans l'espace de tête»). L'essai sur la préparation doit être effectué sur les trois groupes d'espèces (OCDE 201, 202 et 203).

Le tableau 1 récapitule les exigences concernant les différents sous-groupes de produits selon le critère 2.1 .

\section{Évaluation et vérification du critère 2.1}

II convient de fournir à l'organisme compétent des rapports contenant les données sur la toxicité aquatique de la préparation et de tous les composants principaux, obtenues à l'aide de matériel existant tiré d'enregistrements ou de nouveaux essais, et permettant d'attester la conformité avec les exigences du tableau 1.

La toxicité aquatique de la préparation doit être déterminée selon les méthodes d'essai OCDE 201, 202 et 203 ou des méthodes équivalentes. La toxicité aquatique de chaque composant principal doit être déterminée selon les méthodes d'essai OCDE 201 et 202 ou des méthodes équivalentes.

\section{Critère 2.2}

Exigences concernant chaque composant Il convient de fournir des données sur la toxicité aquatique de chaque composant ajouté au produit à dessein. Sont autorisées dans le lubrifiant une ou plusieurs substances présentant un certain degré de toxicité aquatique pour autant que leur concentration en masse cumulée respecte les valeurs indiquées au tableau 2.

\section{Évaluation et vérification du critère $\mathbf{2 . 2}$}

Il convient de fournir à l'organisme compétent des rapports contenant les données sur la toxicité aquatique de chaque composant, obtenues à l'aide de matériel existant tiré d'enregistrements ou de nouveaux essais, et attestant la conformité avec les exigences du tableau 2.

La toxicité aquatique de chaque composant doit être déterminée selon les méthodes d'essai OCDE 201 et 202 ou des méthodes équivalentes.

\section{Évaluation et vérification des deux critères} 2.1 et 2.2

Dans le cas de composants légèrement solubles $(<10 \mathrm{mg} / \mathrm{L})$, il est possible de recourir à la méthode de la fraction adaptée à l'eau (WAF) pour déterminer la toxicité aquatique. Le niveau d'apport fixé, parfois désigné par DL50 et renvoyant à la dose létale, peut être utilisé directement dans les critères de classification. La préparation de la fraction adaptée à l'eau doit respecter les recommandations établies selon l'une des orientations suivantes : rapport technique no 20 (1986) de l'Ecetoc, annexe III de l'essai OCDE 301 1992, ligne directrice 10634 de I'ISO, norme ASTM D6081-98 ("Standard practice for Aquatic Toxicity Tes-

Tableau 1. Exigences en matière de toxicité aquatique concernant les différents sous-groupes de produits. Exigences en matière de données concernant la préparation et ses composants principaux.

\begin{tabular}{|lcccc|}
\hline Critère 2.1 & Fluides hydrauliques & Graisses* & $\begin{array}{c}\text { Huiles pour scies à chaîne, } \\
\text { agents de décoffrage } \\
\text { du béton et autres produits } \\
\text { de graissage d'appoint }\end{array}$ & $\begin{array}{c}\text { Huiles pour moteur } \\
\text { à deux temps }\end{array}$ \\
\hline $\begin{array}{l}\text { Toxicité aquatique du produit } \\
\text { complètement formulé lors des trois essais } \\
\text { de toxicité aiguë OCDE 201, 202 et } 203 \\
\begin{array}{l}\text { Toxicité aquatique de chaque composant } \\
\text { principal lors des essais OCDE 201 et 202 }\end{array}\end{array}$ & $\geq 100 \mathrm{mg} / \mathrm{L}$ & $\geq 1000 \mathrm{mg} / \mathrm{L}$ & $\geq 1000 \mathrm{mg} / \mathrm{L}$ & $\geq 1000 \mathrm{mg} / \mathrm{L}$ \\
\hline
\end{tabular}

* $\|$ est possible de procéder à l'évaluation des graisses de cette façon seulement si l'épaississant présente une biodégradabilité > 70 \% lors de l'essai OCDE 302 C ou selon des méthodes d'essai équivalentes, ou une biodégradabilité $>20 \%$ mais $<60 \%$ après vingt-huit jours lors des essais OCDE fondés sur la déperdition d'oxygène ou la production de gaz carbonique.

Tableau 2. Exigences en matière de toxicité aquatique concernant les différents sous-groupes de produits. Exigences en matière de données concernant chaque composant.

\begin{tabular}{|c|c|c|c|c|}
\hline \multirow{2}{*}{$\begin{array}{l}\text { Critère } 2.2 \\
\text { Toxicité aquatique }\end{array}$} & \multicolumn{4}{|c|}{ Concentration en masse cumulée des substances présentes dans : } \\
\hline & Fluides hydrauliques & Graisses & $\begin{array}{l}\text { Huiles pour scies à chaîne, } \\
\text { agents de décoffrage } \\
\text { du béton et autres produits } \\
\text { de graissage d'appoint }\end{array}$ & $\begin{array}{l}\text { Huiles pour moteur } \\
\text { à deux temps }\end{array}$ \\
\hline $\begin{array}{l}10 \mathrm{mg} / \mathrm{L}<\text { toxicité aiguë }{ }^{*} \leq 100 \mathrm{mg} / \mathrm{L} \\
\text { ou } 1 \mathrm{mg} / \mathrm{L}<\text { CSEO } \leq 10 \mathrm{mg} / \mathrm{L}\end{array}$ & $\leq 20$ & $\leq 25$ & $\leq 5$ & $\leq 25$ \\
\hline $\begin{array}{l}1 \mathrm{mg} / \mathrm{L}<\text { toxicité aiguë* } \leq 10 \mathrm{mg} / \mathrm{L} \\
\text { ou } 0,1 \mathrm{mg} / \mathrm{L}<\mathrm{CSEO} \leq 1 \mathrm{mg} / \mathrm{L}\end{array}$ & $\leq 5$ & $1 \leq$ & $\leq 0,5$ & $\leq 1$ \\
\hline $\begin{array}{l}\text { Toxicité aiguë* }<1 \mathrm{mg} / \mathrm{L} \\
\text { ou CSEO } \leq 0,1 \mathrm{mg} / \mathrm{L}\end{array}$ & $\leq 1$ & $\leq 0,1$ & $\leq 0,1$ & $\leq 0,1$ \\
\hline
\end{tabular}

${ }^{*} \mathrm{CE} 50 / \mathrm{CL} 50 / \mathrm{C} 150$. 
Tableau 3. Exigences en matière de biodégradabilité et de potentiel bioaccumulatif.

\begin{tabular}{|c|c|c|c|c|}
\hline \multirow[b]{2}{*}{ Biodégradation } & \multicolumn{4}{|c|}{ Concentration en masse cumulée des substances } \\
\hline & $\begin{array}{c}\text { Fluides } \\
\text { hydrauliques }\end{array}$ & Graisses & $\begin{array}{l}\text { Huiles pour scies à chaîne, } \\
\text { agents de décoffrage } \\
\text { du béton et autres produits } \\
\text { de graissage d'appoint }\end{array}$ & $\begin{array}{l}\text { Huiles pour moteur } \\
\text { à deux temps }\end{array}$ \\
\hline Non biodégradable* & $\leq 5$ & $\leq 10$ & $\leq 5$ & $\leq 10$ \\
\hline Intrinsèquement biodégradable en milieu aérobie & $\leq 5$ & $\leq 20$ & $\leq 5$ & $\leq 20$ \\
\hline Ultimement biodégradable en milieu aérobie & $\geq 90$ & $\geq 75$ & $\geq 90$ & $\geq 75$ \\
\hline
\end{tabular}

* Remarque : les substances qui sont à la fois non biodégradables et bioaccumulables sont interdites.

ting for Lubricants: Sample Preparation and Results Interpretation or equivalent methods»). II n'est pas nécessaire d'étudier la toxicité aquatique aiguë sur les algues et les daphnies (OCDE 201 et 202):

- s'il est peu probable que la substance traverse des membranes biologiques [MM $>800$ ou diamètre moléculaire > 1,5 $\mathrm{nm}(15 \AA)$ ],

- ou si la substance est fortement insoluble dans l'eau (solubilité dans l'eau $<10 \mu \mathrm{g} / \mathrm{L}$ ), car ces substances ne sont pas considérées comme toxiques pour les algues et les daphnies dans le milieu aquatique.

De même, il n'est pas nécessaire d'étudier la toxicité aquatique aiguë sur les daphnies (OCDE 202) si une étude sur la toxicité à long terme, réalisée selon la méthode d'essai OCDE 211 ou une méthode équivalente, est disponible.

La solubilité des substances dans l'eau doit être déterminée, le cas échéant, selon la méthode d'essai OCDE 105 (ou des méthodes équivalentes).

Si des données sur la toxicité chronique sont disponibles (résultats des essais OCDE 210 et 211 ou de méthodes équivalentes), elles peuvent être utilisées au lieu des données sur la toxicité aquatique aiguë. L'absence de données sur la toxicité chronique doit être établie par écrit à l'aide d'un document signé par le demandeur.

\section{Biodégradabilité et potentiel bioaccumulatif}

Le produit ne doit pas contenir de substances qui sont à la fois :

- non biodégradables,

et

- (potentiellement) bioaccumulables.

Toutefois, le produit peut contenir une ou plusieurs substances présentant un certain degré de dégradabilité et de bioaccumulation potentielle ou réelle pour autant que leur concentration en masse cumulée ne dépasse pas les valeurs indiquées au tableau 3.

\section{Évaluation et vérification du critère 3}

II convient d'attester la conformité avec le critère 3 en fournissant les informations suivantes:
- rapports contenant les données sur la biodégradabilité de chaque composant si cela n'apparaît pas clairement sur les fiches de données de sécurité fournies pour chaque substance,

- rapports contenant les données sur le potentiel bioaccumulatif de chaque composant :

- pour les substances non biodégradables, et

- pour les substances toxiques et très toxiques qui sont facilement biodégradables (aux fins de classification).

La biodégradabilité doit être déterminée séparément pour chaque composant du produit selon les méthodes d'essai indiquées ci-après (ou des méthodes équivalentes).

Une substance est considérée comme ultimement biodégradable (en milieu aérobie) si :

- lors d'une étude de biodégradation de vingthuit jours selon la méthode d'essai OCDE 301 A-F ou des méthodes d'essai équivalentes, les niveaux de biodégradation suivants sont atteints :

- lors d'essais OCDE 301 fondés sur le carbone organique dissous, $\geq 70 \%$;

- lors d'essais OCDE 301 fondés sur la déperdition d'oxygène ou la production de gaz carbonique, $\geq 60 \%$ des maximums théoriques ;

- le rapport DBO5/DThO ou DBO5/DCO est supérieur à 0,5 .

Dans les essais OCDE, le principe de la fenêtre de dix jours ne s'applique pas nécessairement. Si la substance atteint le seuil de biodégradation dans les vingt-huit jours mais pas dans la fenêtre de dix jours, elle est censée avoir une vitesse de dégradation plus lente.

Une substance est considérée comme intrinsèquement biodégradable si l'on constate :

- une biodégradation $>70 \%$ lors de l'essai OCDE $302 C$ de biodégradabilité intrinsèque ou selon une méthode d'essai équivalente, ou - une biodégradation $>20 \%$ mais $<60 \%$ après vingt-huit jours lors des essais OCDE 301 fondés sur la déperdition d'oxygène ou la production de gaz carbonique, ou

- une biodégradation $\geq 60 \%$ lors de l'essai ISO 14593 (essai au CO2 dans l'espace de tête).

Une substance essentiellement destinée à épaissir doit être considérée comme intrinsè- quement biodégradable en milieu aérobie si I'on constate une biodégradation supérieure à $20 \%$ lors de l'essai OCDE 302 C de biodégradabilité intrinsèque ou selon des méthodes d'essai équivalentes. Dans ce cas, les produits de dégradation qui ont été scientifiquement identifiés comme des dérivés de l'épaississant doivent également satisfaire, après exposition dans le milieu aquatique, à toutes les exigences en matière de toxicité aquatique.

Une substance est non biodégradable si elle ne satisfait pas aux critères de biodégradabilité ultime et intrinsèque.

Une substance $\mathrm{n}^{\prime}$ est pas bioaccumulable si sa $\mathrm{MM}$ est $>800$ ou son diamètre moléculaire est $>1,5 \mathrm{~nm}(>15 \AA)$.

Une substance de $\mathrm{MM}<800$ ou de diamètre moléculaire $<1,5 \mathrm{~nm}(<15 \AA)$ n'est pas bioaccumulable si :

- le coefficient de partage octanol/eau (log Kow) est $<3$ ou $>7$, ou

- le FBC mesuré est $\leq 100$. Comme la plupart des substances entrant dans la composition des lubrifiants sont très hydrophobes, la valeur du $\mathrm{FBC}$ doit reposer sur la teneur lipidique en poids et il convient de ménager un temps d'exposition suffisant.

\section{Méthodes d'essai}

Les méthodes à utiliser pour déterminer la biodégradabilité facile sont la méthode OCDE 301 série A-F, les méthodes ISO et ASTM équivalentes ou le rapport DBO5/(DThO ou DCO). Le rapport DBO5/(DThO ou DCO) ne peut être utilisé que s'il n'existe aucune donnée disponible sur la base de la méthode d'essai OCDE 301 ou de méthodes d'essai équivalentes. La DBO5 doit être évaluée selon la méthode C.5 [directive 92/69/CEE de la Commission ${ }^{5}$ ] ou des méthodes équivalentes, tandis que la DCO doit être évaluée selon la méthode C.6 (directive $92 / 69 /$ CEE) ou des méthodes équivalentes. Pour déterminer la biodégradabilité intrinsèque, il convient d'utiliser la méthode d'essai OCDE 302 C ou des méthodes d'essai équivalentes.

\footnotetext{
$\overline{5}$ JO L 383 du 29.12.1992, p. 113.
} 
Le demandeur peut également utiliser des références croisées afin d'évaluer la biodégradabilité d'une substance. Les références croisées pour l'évaluation de la biodégradabilité d'une substance seront acceptées si la substance de référence ne diffère de la substance utilisée dans le produit que par un seul groupe ou fragment fonctionnel. Si la substance de référence est facilement ou intrinsèquement biodégradable et si le groupe fonctionnel a un effet positif sur la biodégradation aérobie, la substance utilisée peut aussi être considérée comme facilement ou intrinsèquement biodégradable. Les groupes ou fragments fonctionnels ayant un effet positif sur la biodégradation sont les suivants : alcool aliphatique et aromatique $[-\mathrm{OH}]$, acide aliphatique et aromatique $[-\mathrm{C}(=\mathrm{O})-\mathrm{OH}]$, aldéhyde [-CHO], ester $[-\mathrm{C}(=\mathrm{O})$ $\mathrm{O}-\mathrm{C}]$, amide $[-\mathrm{C}(=\mathrm{O})-\mathrm{N}$ ou $-\mathrm{C}(=\mathrm{S})-\mathrm{N}]$. II convient de fournir une documentation appropriée et fiable concernant l'étude sur la substance de référence. En cas de comparaison avec un fragment non mentionné ci-dessus, il convient de fournir une documentation appropriée et fiable concernant les études sur l'effet positif du groupe fonctionnel sur la biodégradation de substances de structure similaire. Le coefficient de partage octanol/eau (log Kow) doit être évalué selon les méthodes d'essai OCDE 107, 117, le projet de méthode 123, ou toute autre méthode d'essai équivalente. Le facteur de bioconcentration (FBC) doit être évalué selon la méthode d'essai OCDE 305.

Les valeurs du log Kow ne concernent que les produits chimiques organiques. Pour évaluer le potentiel bioaccumulatif de composés inorganiques, de certains tensioactifs et composés organométalliques, il convient d'effectuer des mesures du FBC.

Si l'essai ne peut être effectué (par exemple si la substance a une activité superficielle importante ou ne se dissout pas dans l'eau ni l'octanol), il convient de fournir une valeur calculée du log Kow ainsi que les détails de la méthode de calcul. Pour le calcul du log Kow, sont autorisées les méthodes suivantes: CLOGP pour un log Kow compris entre 0 et 9, LOGKOW (KOWWIN) pour un log Kow compris entre - 4 et 8 , AUTOLOGP pour un log Kow supérieur à 5 , comme indiqué dans le règle- ment (CE) no 1488/94 de la Commission ${ }^{6}$ étayé par un document d'orientation technique.

\section{Exclusion de substances spécifiques}

Aucune des substances figurant dans la liste communautaire des substances prioritaires dans le domaine de l'eau et dans la liste OSPAR de produits chimiques devant faire l'objet de mesures prioritaires, dans leur version applicable en décembre 2004, ne doit être ajoutée à dessein dans la composition d'un produit éligible au label écologique communautaire.

Aucun composé organohalogéné ni nitrique ne doit être ajouté à dessein dans la composition d'un produit éligible au label écologique communautaire.

Aucun métal ni composé métallique ne doit être ajouté à dessein dans la composition d'un produit éligible au label écologique communautaire, à l'exception du sodium, du potassium, du magnésium et du calcium. Dans le cas des épaississants, les composés du lithium et/ou de l'aluminium peuvent aussi être utilisés à des concentrations respectant les autres critères de la présente annexe.

\section{Évaluation et vérification du critère 4}

Le respect de ces exigences doit être établi par écrit à l'aide d'un document signé par le demandeur.

\section{Matières premières recyclables}

Le produit formulé doit avoir une teneur en carbone provenant de matières premières recyclables de:

$-\geq 50 \%(\mathrm{~m} / \mathrm{m})$ pour les huiles hydrauliques ; $-\geq 45 \%(\mathrm{~m} / \mathrm{m})$ pour les graisses;

$-\geq 70 \%(\mathrm{~m} / \mathrm{m})$ pour les huiles pour scies à chaîne, agents de décoffrage du béton et autres produits de graissage d'appoint ;

$-\geq 50 \%(\mathrm{~m} / \mathrm{m})$ pour les huiles pour moteur à deux temps.

Par teneur en carbone provenant de matières premières recyclables, on entend le pourcentage en masse du composant $\mathrm{A}^{\circ}-$ [nombre d'atomes de carbone dans le composant A provenant $d$ 'huiles (végétales) ou de graisses

${ }^{6}$ JO L 161 du 29.6.1994, p. 3. (animales) divisé par le nombre total d'atomes de carbone dans le composant $A]$ plus le pourcentage en masse du composant $B^{\circ}-$ [nombre d'atomes de carbone dans le composant $B$ provenant d'huiles (végétales) ou de graisses (animales) divisé par le nombre total d'atomes de carbone dans le composant B] plus le pourcentage en masse du composant $C^{\circ}-$ [nombre $\mathrm{d}^{\prime}$ atomes de carbone dans le composant $\mathrm{C}$ ] et ainsi de suite.

\section{Évaluation et vérification du critère 5}

Le demandeur doit fournir à l'organisme compétent une déclaration de conformité avec ce critère.

\section{Performance technique}

Les huiles hydrauliques doivent au moins satisfaire aux critères de performance technique établis dans la norme ISO 15380, tableaux 2, 3. Les graisses doivent être « adaptées à l'usage prévu ».

Les huiles pour scies à chaîne doivent au moins satisfaire aux critères de performance technique établis dans la norme RAL-UZ 48 de I'Ange Bleu.

Les agents de décoffrage du béton et autres produits de graissage d'appoint doivent être adaptés à l'usage prévu.

Les huiles pour moteur à deux temps doivent au moins satisfaire aux critères de performance technique établis dans le document «NMMA Certification for Two-Stroke Cycle Gasoline Engine Lubricants» de NMMA TC-W3.

Évaluation et vérification du critère 6

Le demandeur doit fournir à l'organisme compétent une déclaration de conformité avec ce critère ainsi que la documentation correspondante.

\section{Informations figurant sur le label écologique}

Le cadre 2 du label écologique doit contenir le texte suivant: "utilisation peu nocive pour l'eau et le sol ; émissions de $\mathrm{CO}_{2}$ limitées ».

\section{Évaluation et vérification du critère 7}

Le demandeur doit fournir à l'organisme compétent un échantillon de l'emballage du produit faisant apparaître le label ainsi qu'une déclaration de conformité avec ce critère. 